\title{
Oblivious transfer using quantum entanglement
}

\author{
Guang Ping He \\ Department of Physics, The University of Hong Kong, Pokfulam Road, Hong Kong, China and \\ Department of Physics \& Advanced Research Center, \\ Zhongshan University, Guangzhou \\ 510275, China \\ Z. D. Wang \\ Department of Physics 83 Center of Theoretical and Computational Physics, \\ The University of Hong Kong, Pokfulam Road, Hong Kong, China and \\ National Laboratory of Solid State Microstructures, Nanjing University, Nanjing 210093, China
}

\begin{abstract}
Based on quantum entanglement, an all-or-nothing oblivious transfer protocol is proposed and is proven to be secure. The distinct merit of the present protocol lies in that it is not based on quantum bit commitment. More intriguingly, this OT protocol does not belong to a class of protocols denied by the Lo's no-go theorem of one-sided two-party secure computation, and thus its security can be achieved.
\end{abstract}

PACS numbers: 03.67.Dd, 03.67.Hk, 03.67.Mn, 89.70.+c

\section{INTRODUCTION}

The research on the oblivious transfer (OT) problem may be traced back to more than twenty years ago 1, 2]. Kilian [3] pointed out later that OT is very important in two-party and multi-party protocols. This is because in most symmetrical protocols, the participants always know each others' data. If some participants are dishonest or try to get extra information, the protocols become insecure. OT can create some secret between the participants and break this symmetry. Thus it can be used to implement multi-party protocols such as two-party secure computation [4]. However, the security of classical cryptography usually has to be based on some strong computational assumptions, such as the hardness of factoring. If quantum computers become practical in the future, the validity of these assumptions can be broken easily [5]. Therefore significant interests have been paid to look for quantum methods applicable to cryptography to achieve better security [6, 7]. Quantum oblivious transfer (QOT) protocols were also proposed [8]. But they are secure only under the assumption that the participants cannot delay the quantum measurement. To fix the problem, Crépeau $[9]$ proposed a QOT protocol based on quantum bit commitment (QBC). It was further proven by Yao 10 that such a QOT is secure if QBC is secure. Nevertheless, it was indicated later by Mayers, Lo and Chau that all the QBC protocols formerly proposed [6, 11] are insecure. Furthermore, it was concluded that unconditionally secure QBC scheme cannot be achieved in principle 12, 13, which is referred to as the Mayers-Lo-Chau (MLC) no-go theorem and is a serious drawback in quantum cryptography. According to the theorem, all QBC based protocols are insecure, including quantum coin tossing $[6,14,15,16]$ and quantum oblivious mutual identification [17. Consequently, QOT based on QBC is insecure unless the participants are restricted to individual measurements 18.
On the other hand, starting with QBC is not the only way to implement QOT. Therefore, it is natural to ask whether we can design a QOT protocol with stand-alone security. Although it was concluded independently that other two-party quantum secure computations including QOT are not possible either [19, 20], the conclusion is essentially based on a crucial point that the quantum state used in the two-party computation protocols is the simultaneous eigenstate of different measurement operators, which follows from two basic requirements in their definition of the so-called ideal one-sided two-party secure computation [19]: Alice helps Bob to compute a prescribed function $f(i, j)$ in such a way that, at the end of the protocol, (a) Bob learns $f(i, j)$ unambiguously, and (b) Alice learns nothing. In this paper, a novel quantum OT protocol is proposed, which is neither based on QBC nor satisfying rigorously the requirement (a); but it indeed meets the rigorous security requirement of the OT definition. Therefore, our OT protocol is a kind of two-party secure computation different from that defined by Lo in Ref. [19] and thus evades the Lo's no-go theorem of the one-sided two-party secure computation, allowing more quantum-cryptography applications than thought possible previously.

In the next section, a new QOT protocol is elaborated in details. Then a general proof of its unconditional security is presented in Sec. III. Finally, the relationship between the protocol and the no-go theorems is addressed.

\section{THE SCHEME}

Although there are various types of OT, as a typical illustration, we here focus only on a basic type OT studied in Refs. 1, 10], which is also called all-or-nothing OT. A sender Alice wants to transfer a secret bit $b \in\{0,1\}$ to a receiver Bob. At the end of the protocol, either Bob could learn the value of $b$ with the reliability (which means the 
probability for Bob's output $b$ to be equal to Alice's input) $100 \%$, or he has zero knowledge on $b$. Each possibility should occur with the probability $1 / 2$, and which one happens finally is out of their control. Meanwhile, Alice should learn nothing about which event takes place.

Consider an ideal case without transmission error. Similar to the conjugate coding [2], letting $|0\rangle_{+}$and $|1\rangle_{+}$ denote the two orthogonal states of a qubit, we can define $|r\rangle_{\times} \equiv\left(|0\rangle_{+}+(-1)^{r}|1\rangle_{+}\right) / \sqrt{2}(r=0,1)$, the Bell states $\Phi^{ \pm} \equiv\left(|0\rangle_{+}|0\rangle_{+} \pm|1\rangle_{+}|1\rangle_{+}\right) / \sqrt{2}$, and $\Psi^{ \pm} \equiv$ $\left(|0\rangle_{+}|1\rangle_{+} \pm|1\rangle_{+}|0\rangle_{+}\right) / \sqrt{2}$, where $+(\times)$ stands for the rectilinear (diagonal) basis. The key idea of our protocol is: Alice and Bob share many sets of 4 qubits in an entangled state $|\psi\rangle$ (see Eq. (1) below). To each set, four two-value parameters $q, r, c$, and $d$ are associated, where $\{q, r\}$ and $\{c, d\}$ correspond respectively to the state $|\psi\rangle$ and the choice/measurement of individual participant; the form of $|\psi\rangle$ designed by us ensures that Alice cannot decode simultaneously any two of $q, r$ and $d$, and Bob cannot decode $c$ and $q$ (or $r$ ) simultaneously. Relying on appropriate verification and use of state, a secure OT can be achieved.

For easy readability, before presenting a complete version of our protocol, we first account for the details in several key procedures comprehensibly.

(i) Preparation of the states:

Our protocol is based on the four-qubit entangled state with the following form

$$
\begin{aligned}
|\psi\rangle= & \left|\psi_{A_{1}} \psi_{A_{2}} \psi_{B_{1}} \psi_{B_{2}}\right\rangle \\
= & \left(|0\rangle_{+}|0\rangle_{+}|0\rangle_{+}|0\rangle_{+}\right. \\
& +|1\rangle_{+}|1\rangle_{+}|0\rangle_{+}|1\rangle_{+} \\
& +|0\rangle_{\times}|0\rangle_{\times}|1\rangle_{+}|0\rangle_{+} \\
& \left.+|1\rangle_{\times}|1\rangle_{\times}|1\rangle_{+}|1\rangle_{+}\right) / 2 .
\end{aligned}
$$

Bob prepares many sets of such states. For each set, he keeps systems $B_{1}$ and $B_{2}$ and sends systems $A_{1}$ and $A_{2}$ to Alice.

(ii) Alice inputting $c$ :

In Alice's point of view, Bob sends her any of the four two-qubit states $|r\rangle_{q}|r\rangle_{q}(q \in\{+, \times\}, r \in\{0,1\})$ with the equal probability. Now let us consider Alice's strategy to decode either $q$ or $r$. In the Bell basis

$$
C_{0} \equiv\left\{\Phi^{+}, \Phi^{-}, \Psi^{+}, \Psi^{-}\right\}
$$

the four possible $|r\rangle_{q}|r\rangle_{q}$ can be expressed as

$$
\begin{aligned}
& |0\rangle_{+}|0\rangle_{+}=\left(\Phi^{+}+\Phi^{-}\right) / \sqrt{2} \\
& |1\rangle_{+}|1\rangle_{+}=\left(\Phi^{+}-\Phi^{-}\right) / \sqrt{2} \\
& |0\rangle_{\times}|0\rangle_{\times}=\left(\Phi^{+}+\Psi^{+}\right) / \sqrt{2} \\
& |1\rangle_{\times}|1\rangle_{\times}=\left(\Phi^{+}-\Psi^{+}\right) / \sqrt{2}
\end{aligned}
$$

If Alice measures systems $A_{1}$ and $A_{2}$ in the $C_{0}$ basis, she will know that $q=+(q=\times)$ if the outcome is $\Phi^{-}\left(\Psi^{+}\right)$.
While if the outcome is $\Phi^{+}$, she will not know the value of $q$. Since Eq. (1) can be rewritten as

$$
\begin{aligned}
& |\psi\rangle=\Phi^{-}|0\rangle_{+}|1\rangle_{\times} / 2+\Psi^{+}|1\rangle_{+}|1\rangle_{\times} / 2 \\
& +\Phi^{+}|0\rangle_{\times}|0\rangle_{\times} \quad / \sqrt{2}
\end{aligned}
$$

it can be seen that the probability for Alice to decode $q$ successfully is $1 / 2$.

On the other hand, defining the basis

$$
C_{1} \equiv\left\{|0\rangle_{\times}|0\rangle_{+},|0\rangle_{\times}|1\rangle_{+},|1\rangle_{\times}|0\rangle_{+},|1\rangle_{\times}|1\rangle_{+}\right\},
$$

$|r\rangle_{q}|r\rangle_{q}$ can be expressed as

$$
\begin{aligned}
|0\rangle_{+}|0\rangle_{+} & =\left(|0\rangle_{\times}|0\rangle_{+}+|1\rangle_{\times}|0\rangle_{+}\right) / \sqrt{2} \\
|1\rangle_{+}|1\rangle_{+} & =\left(|0\rangle_{\times}|1\rangle_{+}-|1\rangle_{\times}|1\rangle_{+}\right) / \sqrt{2} \\
|0\rangle_{\times}|0\rangle_{\times} & =\left(|0\rangle_{\times}|0\rangle_{+}+|0\rangle_{\times}|1\rangle_{+}\right) / \sqrt{2} \\
|1\rangle_{\times}|1\rangle_{\times} & =\left(|1\rangle_{\times}|0\rangle_{+}-|1\rangle_{\times}|1\rangle_{+}\right) / \sqrt{2}
\end{aligned}
$$

That is, if Alice measures them in the $C_{1}$ basis, she will know that $r=0(r=1)$ if the outcome is $|0\rangle_{\times}|0\rangle_{+}$ $\left(|1\rangle_{\times}|1\rangle_{+}\right)$, while she does not know $r$ if the outcome is $|0\rangle_{\times}|1\rangle_{+}$or $|1\rangle_{\times}|0\rangle_{+}$. Again, rewriting Eq.(1) as

$$
\begin{aligned}
|\psi\rangle= & |0\rangle_{\times}|0\rangle_{+}|0\rangle_{\times}|0\rangle_{+} / 2-|1\rangle_{\times}|1\rangle_{+}|0\rangle_{\times}|1\rangle_{+} / 2 \\
& +|0\rangle_{\times}|1\rangle_{+} \Psi^{+} / 2+|1\rangle_{\times}|0\rangle_{+} \Phi^{+} / 2
\end{aligned}
$$

we see that the probability for Alice to decode $r$ successfully is also $1 / 2$. Also, since the bases $C_{0}$ and $C_{1}$ are not commutable, Alice cannot decode the values of $q$ and $r$ simultaneously (A rigorous proof will be provided in the next section).

In our protocol, Alice should randomly picks a different bit $c \in\{0,1\}$ for each set of $|\psi\rangle$ at this stage. If $c=0$ $(c=1)$, she tries to decode $q(r)$ by measuring her share of the set in the $C_{0}\left(C_{1}\right)$ basis. After she measures all $|\psi\rangle$, she will decode either $q$ or $r$ successfully for about half of these sets, while she fails to decode anything for the other half. She tells Bob to discard the half which she failed to decode, while keeps the rest sets of $|\psi\rangle$ in the following steps.

Bob can verify whether Alice has input $c$ and finished her measurement by picking randomly some $|\psi\rangle$ from the remaining half, and asking Alice to announce either $q$ or $r$, depending on what she decoded. To find out the correct value of $q$ or $r$, as can be seen from Eq.(1), Bob can simply measures systems $B_{1}$ and $B_{2}$ of the picked $|\psi\rangle$ in the basis

$$
D_{0} \equiv\left\{|0\rangle_{+}|0\rangle_{+},|0\rangle_{+}|1\rangle_{+},|1\rangle_{+}|0\rangle_{+},|1\rangle_{+}|1\rangle_{+}\right\} .
$$

Then he learns which $|r\rangle_{q}|r\rangle_{q}$ systems $A_{1}$ and $A_{2}$ can collapse to. If Alice has delayed her measurement or adopted any other measurement which is less efficient than the above strategies on decode $q$ or $r$ with certainty, she cannot always announce $q$ or $r$ correctly, or she has to discard more than half of the sets. Therefore a dishonest Alice will inevitably be caught as the increase of the number of $|\psi\rangle$ picked for the verification. 
Nevertheless, to pass the verification, Alice needs not to perform complete measurement in the $C_{0}$ $\left(C_{1}\right)$ basis. She can simply try to project systems $A_{1}$ and $A_{2}$ to the subspace supported by $\left\{\Phi^{-}, \Psi^{+}\right\}$ $\left(\left\{|0\rangle_{\times}|0\rangle_{+},|1\rangle_{\times}|1\rangle_{+}\right\}\right)$. If the projection fails, she tells Bob to discard the corresponding $|\psi\rangle$. While if the projection is successful, she keeps systems $A_{1}$ and $A_{2}$ entangled with $B_{1}$ and $B_{2}$ without collapsing them into a pure state $\Phi^{-}$or $\Psi^{+}\left(|0\rangle_{\times}|0\rangle_{+}\right.$or $\left.|1\rangle_{\times}|1\rangle_{+}\right)$. She finishes the complete measurement to make them collapse only when the corresponding $|\psi\rangle$ is picked for the verification. Therefore in general, the state of the remaining undiscarded and unverified sets of $|\psi\rangle$ is either

$$
\left|\psi^{(0)}\right\rangle=\Phi^{-}|0\rangle_{+}|1\rangle_{\times} / \sqrt{2}+\Psi^{+}|1\rangle_{+}|1\rangle_{\times} / \sqrt{2}
$$

if $c=0$, or

$$
\left|\psi^{(1)}\right\rangle=|0\rangle_{\times}|0\rangle_{+}|0\rangle_{\times}|0\rangle_{+} / \sqrt{2}-|1\rangle_{\times}|1\rangle_{+}|0\rangle_{\times}|1\rangle_{+} / \sqrt{2}
$$

if $c=1$. After the verification, Alice and Bob keep these $|\psi\rangle$ and proceed.

(iii) Bob inputting d:

Since the state of systems $B_{1}$ and $B_{2}$ are different in Eqs.(9) and (10), Bob can learn Alice's choice of $c$ or her outcome $s$ with a certain probability. Here Alice's outcome $s$ is defined as

$$
s \equiv \begin{cases}Q, & (c=0), \\ r, & (c=1),\end{cases}
$$

where $Q=0,1$ for $q=+, \times$. From Eq. (11) we can see that if Bob measures systems $B_{1}$ and $B_{2}$ in the $D_{0}$ basis defined in Eq.(8) and the outcome is $|0\rangle_{+}|0\rangle_{+}$(or $|1\rangle_{+}|1\rangle_{+}$), he will know that systems $A_{1}$ and $A_{2}$ can only collapse to the state $|0\rangle_{+}|0\rangle_{+}\left(\right.$or $\left.|1\rangle_{\times}|1\rangle_{\times}\right)$. These two states have the common feature $Q=r$. Thus Bob knows that $s=0(s=1)$ despite he does not know $c$.

Note that at this stage, $|\psi\rangle$ already collapsed to $\left|\psi^{(0)}\right\rangle$ or $\left|\psi^{(1)}\right\rangle$ by Alice's measurement. With the $D_{0}$ basis, they can be expressed as

$$
\begin{aligned}
\left|\psi^{(0)}\right\rangle= & {\left[\Phi^{-}\left(|0\rangle_{+}|0\rangle_{+}-|0\rangle_{+}|1\rangle_{+}\right)\right.} \\
& \left.+\Psi^{+}\left(|1\rangle_{+}|0\rangle_{+}-|1\rangle_{+}|1\rangle_{+}\right)\right] / 2,
\end{aligned}
$$

and

$$
\begin{aligned}
\left|\psi^{(1)}\right\rangle= & {\left[|0\rangle_{\times}|0\rangle_{+}\left(|0\rangle_{+}|0\rangle_{+}+|1\rangle_{+}|0\rangle_{+}\right)\right.} \\
& \left.-|1\rangle_{\times}|1\rangle_{+}\left(|0\rangle_{+}|1\rangle_{+}+|1\rangle_{+}|1\rangle_{+}\right)\right] / 2 .
\end{aligned}
$$

Thus the probability for Bob to decode $s$ successfully is $1 / 2$.

On the other hand, defining the basis

$$
D_{1} \equiv\left\{|0\rangle_{\times}|0\rangle_{\times},|0\rangle_{\times}|1\rangle_{\times},|1\rangle_{\times}|0\rangle_{\times},|1\rangle_{\times}|1\rangle_{\times}\right\},
$$

$\left|\psi^{(0)}\right\rangle$ or $\left|\psi^{(1)}\right\rangle$ can be expressed as

$$
\left|\psi^{(0)}\right\rangle=\left(\Phi^{-}-\Psi^{+}\right)|1\rangle_{\times}|1\rangle_{\times} / 2+\left(\Phi^{-}+\Psi^{+}\right)|0\rangle_{\times}|1\rangle_{\times} / 2,
$$

and

$$
\begin{aligned}
\left|\psi^{(1)}\right\rangle= & \left(|0\rangle_{\times}|0\rangle_{+}-|1\rangle_{\times}|1\rangle_{+}\right)|0\rangle_{\times}|0\rangle_{\times} / 2 \\
& +\left(|0\rangle_{\times}|0\rangle_{+}+|1\rangle_{\times}|1\rangle_{+}\right)|0\rangle_{\times}|1\rangle_{\times} / 2 .
\end{aligned}
$$

If Bob measures systems $B_{1}$ and $B_{2}$ in the $D_{1}$ basis, he will know that $c=0(c=1)$ if the outcome is $|1\rangle_{\times}|1\rangle_{\times}$ $\left(|0\rangle_{\times}|0\rangle_{\times}\right)$, while he does not know $c$ if the outcome is $|0\rangle_{x}|1\rangle_{\times}$. The probability for him to decode $c$ successfully is also 1/2. Again, Bob cannot decode the values of $s$ and $c$ simultaneously since the bases $D_{0}$ and $D_{1}$ are not commutable.

In the protocol, Bob randomly picks a different bit $d \in\{0,1\}$ for each remaining set of $|\psi\rangle$, where $d=0$ should occur with the probability $p=2 / 3$ (we will see later why this value is chosen). If $d=0(d=1)$, he tries to decode $s(c)$ by measuring his share of the set in the $D_{0}\left(D_{1}\right)$ basis. After he measures all sets of $|\psi\rangle$, he will decode either $s$ or $c$ successfully for about half of those sets, while he fails to decode anything for the other half. He tells Alice to discard the half which he failed to decode, while keeping the rest $|\psi\rangle$ for the following steps.

Similar to (ii), Alice can verify whether Bob has input $d$ and finished his measurement honestly by picking randomly some $|\psi\rangle$ from the remaining half, and asking Bob to announce either $s$ or $c$, depending on what he decoded. She should also check whether Bob has indeed input $d=0$ with the required probability $p=2 / 3$, and whether the number of discarded $|\psi\rangle$ is about a half.

Also, Bob needs not to perform a complete measurement in the $D_{0}\left(D_{1}\right)$ basis to pass the verification. If he has chosen $d=0(d=1)$, he simply tries to project systems $B_{1}$ and $B_{2}$ to the subspace supported by $\left\{|0\rangle_{+}|0\rangle_{+},|1\rangle_{+}|1\rangle_{+}\right\} \quad\left(\left\{|0\rangle_{\times}|0\rangle_{\times},|1\rangle_{\times}|1\rangle_{\times}\right\}\right)$, and discards $|\psi\rangle$ if the projection fails. He finishes the complete measurement to make the undiscarded $|\psi\rangle$ collapse only when it is picked for the verification. Therefore after the verification, the state of the remaining unverified $|\psi\rangle$ is

$$
\left|\psi^{(00)}\right\rangle=\left(\Phi^{-}|0\rangle_{+}|0\rangle_{+}-\Psi^{+}|1\rangle_{+}|1\rangle_{+}\right) / \sqrt{2}
$$

if $c=0$ and $d=0$, or

$$
\left|\psi^{(10)}\right\rangle=\left(|0\rangle_{\times}|0\rangle_{+}|0\rangle_{+}|0\rangle_{+}-|1\rangle_{\times}|1\rangle_{+}|1\rangle_{+}|1\rangle_{+}\right) / \sqrt{2}
$$

if $c=1$ and $d=0$, or

$$
\left|\psi^{(01)}\right\rangle=\left(\Phi^{-}-\Psi^{+}\right)|1\rangle_{\times}|1\rangle_{\times} / \sqrt{2},
$$

if $c=0$ and $d=1$, or

$$
\left|\psi^{(11)}\right\rangle=\left(|0\rangle_{\times}|0\rangle_{+}-|1\rangle_{\times}|1\rangle_{+}\right)|0\rangle_{\times}|0\rangle_{\times} / \sqrt{2},
$$

if $c=1$ and $d=1$.

Before using these states for the OT, Bob must prevent Alice from knowing his choice of $d$ for each of them. It 
can be accomplished with the following method. $\left|\psi^{(00)}\right\rangle$ and $\left|\psi^{(10)}\right\rangle$ can be rewritten as

$$
\left|\psi^{(00)}\right\rangle=\left(\Phi^{-}+\Psi^{+}\right) \Phi^{-} / 2+\left(\Phi^{-}-\Psi^{+}\right) \Phi^{+} / 2,
$$

and

$$
\begin{aligned}
\left|\psi^{(10)}\right\rangle= & \left(|0\rangle_{\times}|0\rangle_{+}+|1\rangle_{\times}|1\rangle_{+}\right) \Phi^{-} / 2 \\
& +\left(|0\rangle_{\times}|0\rangle_{+}-|1\rangle_{\times}|1\rangle_{+}\right) \Phi^{+} / 2 .
\end{aligned}
$$

If Bob has chosen $d=0$, he tries to project systems $B_{1}$ and $B_{2}$ to the state $\Phi^{+}$, and tells Alice to discard the corresponding $|\psi\rangle$ if the projection fails. Then the remaining $\left|\psi^{(00)}\right\rangle$ and $\left|\psi^{(10)}\right\rangle$ collapse to

$$
\left|\psi^{(00 a)}\right\rangle=\left(\Phi^{-}-\Psi^{+}\right) \Phi^{+} / \sqrt{2},
$$

and

$$
\left|\psi^{(10 a)}\right\rangle=\left(|0\rangle_{\times}|0\rangle_{+}-|1\rangle_{\times}|1\rangle_{+}\right) \Phi^{+} / \sqrt{2} .
$$

We can see that the states of systems $A_{1}$ and $A_{2}$ of $\left|\psi^{(00 a)}\right\rangle$ and $\left|\psi^{(10 a)}\right\rangle$ (which are corresponding to $d=0$ ) are exactly the same as those of $\left|\psi^{(01)}\right\rangle$ and $\left|\psi^{(11)}\right\rangle$ (corresponding to $d=1$ ) respectively. Therefore Alice can by no means distinguish them apart so she cannot know $d$ from the remaining $|\psi\rangle$.

Now let us explain why Bob should choose $d=0$ with the probability $p=2 / 3$. Eqs. (21) and (22) show that half of the $|\psi\rangle$ corresponding to $d=0$ will be further discarded when collapsing $\left|\psi^{(00)}\right\rangle$ and $\left|\psi^{(10)}\right\rangle$ to $\left|\psi^{(00 a)}\right\rangle$ and $\left|\psi^{(10 a)}\right\rangle$. Meanwhile, no $|\psi\rangle$ corresponding to $d=1$ will be discarded after Alice has verified Bob's action. Therefore among all the remaining $|\psi\rangle, d=0$ and $d=1$ will occur with the equal probability $1 / 2$, which will be useful below.

(iv) Completing the OT:

At this stage, for any remaining $|\psi\rangle$, Alice knows her own choice $c$ but not Bob's choice $d$, while Bob has chosen $d=0$ (i.e. he does not knows $c$ ) and $d=1$ (he knows $c$ ) with the equal probability $1 / 2$. Thus Alice can randomly pick any one of the remaining $|\psi\rangle$, and use $c$ to encode the bit $b$ she wants to transfer. If by chance Bob knows $c$ for this chosen $|\psi\rangle$, he can decode $b$ successfully. Else he knows nothing about $b$. Because the two results will occur with the equal probability $1 / 2$, the goal of OT is accomplished.

The above procedure is summarized as the protocol below, with the corresponding schematic flow chart being illustrated in Fig.1.

\section{Protocol OT}

(1) Preparation of the states: Bob prepares $n$ sets of $|\psi\rangle$ as described in Eq.(11). He keeps systems $B_{1}$ and $B_{2}$ of each $|\psi\rangle$ and sends systems $A_{1}$ and $A_{2}$ to Alice;

(2) Alice inputting $c$ :
(2-1) For each $|\psi\rangle$, Alice views the state of systems $A_{1}$ and $A_{2}$ as $|r\rangle_{q}|r\rangle_{q}$, and she randomly picks $c \in\{0,1\}$. If $c=0$, She tries to decode $q$ by projecting the two qubits into $\Phi^{-}$and $\Psi^{+}$, and she sets $q=+(q=\times)$ if the outcome is $\Phi^{-}\left(\Psi^{+}\right)$. Else if $c=1$, Alice tries to decode $r$ by projecting the two qubits into $|0\rangle_{\times}|0\rangle_{+}$and $|1\rangle_{\times}|1\rangle_{+}$, and she sets $r=0(r=1)$ if the outcome is $|0\rangle_{\times}|0\rangle_{+}\left(|1\rangle_{\times}|1\rangle_{+}\right)$

(2-2) If the projection in (2-1) fails, Alice tells Bob to discard the corresponding $|\psi\rangle$;

(3) Verification 1:

(3-1) If the number of the remaining $|\psi\rangle$ is $n^{\prime} \backsim$ $n / 2$ they continue 21], else they abort the procedure;

(3-2) Bob randomly picks some of the remaining $|\psi\rangle$ and asks Alice to announce either their $q$ or $r$ depending on the value of $c$. To check Alice's announcement, Bob measures $\psi_{B_{1}} \psi_{B_{2}}$ in the $D_{0}$ basis, and uses the result to calculate $q, r$ that corresponds to $\psi_{A_{1}} \psi_{A_{2}}$;

(3-3) Alice randomly picks some other remaining $|\psi\rangle$ and asks Bob to announce both $q$ and $r$. Bob performs the same measurement in (3-2) to obtain $q, r$ to announce;

(3-4) If \{no conflicting results were found by both participants\} AND the probabilities for $|r\rangle_{q}|r\rangle_{q}=$ $|0\rangle_{+}|0\rangle_{+},|r\rangle_{q}|r\rangle_{q}=|1\rangle_{+}|1\rangle_{+},|r\rangle_{q}|r\rangle_{q}=|0\rangle_{\times}|0\rangle_{\times}$and $|r\rangle_{q}|r\rangle_{q}=|1\rangle_{\times}|1\rangle_{\times}$to occur are approximately the same\}, they keep the remaining undiscarded and unverified $|\psi\rangle$ and continue;

(4) Bob inputting d:

(4-1) For each of the remaining $m$ sets of $|\psi\rangle$, Bob picks $d=0$ with the probability $p=2 / 3$ and $d=1$ with the probability $(1-p)=1 / 3$. If $d=0$, he tries to decode $s$ (defined as Eq.(11)) by projecting $\psi_{B_{1}} \psi_{B_{2}}$ into the subspace supported by $\left\{|0\rangle_{+}|0\rangle_{+},|1\rangle_{+}|1\rangle_{+}\right\}$. Else if $d=1$, Bob tries to decode $c$ by projecting $\psi_{B_{1}} \psi_{B_{2}}$ into $|1\rangle_{\times}|1\rangle_{\times}$and $|0\rangle_{\times}|0\rangle_{\times}$. If the outcome is $|1\rangle_{\times}|1\rangle_{\times}$ $\left(|0\rangle_{\times}|0\rangle_{\times}\right)$, he knows that Alice has chosen $c=0(c=1)$;

(4-2) If the projection in (4-1) fails, Bob tells Alice to discard the corresponding $|\psi\rangle$;

(5) Verification 2:

(5-1) If the number of the remaining $|\psi\rangle$ is about $m / 2$ they continue; else they abort the procedure;

(5-2) Alice randomly picks some of the remaining $|\psi\rangle$ and asks Bob to announce either $c$ or $s$ depending on the value of $d$. Note that if $d=0$, Bob needs to complete the measurement on $\psi_{B_{1}} \psi_{B_{2}}$ in the basis $\left\{|0\rangle_{+}|0\rangle_{+},|1\rangle_{+}|1\rangle_{+}\right\}$, and he announces $s=0(s=1)$ if the outcome is $|0\rangle_{+}|0\rangle_{+}\left(|1\rangle_{+}|1\rangle_{+}\right)$;

(5-3) If no conflicting results were found $A N D$ $\{d=0$ occurs with the probability $2 / 3\}$, they keep the remaining undiscarded and unverified $|\psi\rangle$ and continue;

(6) Bob preventing Alice from knowing d: For each remaining $|\psi\rangle$ which Bob has chosen $d=0$, he tries to project $\psi_{B_{1}} \psi_{B_{2}}$ into the state $\Phi^{+}$, and tells Alice to discard the corresponding $|\psi\rangle$ if the projection fails;

(7) OT part:

(7-1) Alice randomly picks one of the remaining $|\psi\rangle$ and tells Bob $b^{\prime}=b \oplus c$; 
(7-2) If Bob has chosen $d=1$ for this $|\psi\rangle$ he calculates $b=b^{\prime} \oplus c$. Else he knows that he fails to get $b$.

\section{PROOF OF SECURITY}

We now prove generally that the protocol is secure against any cheating strategy in three steps: (I) the form of $|\psi\rangle$ limits the knowledge of Alice and Bob; (II) the verifications limit both participants' behaviors to honest ones; and (III) if Bob does not prepare $|\psi\rangle$ honestly, his knowledge on the data will be even worse.

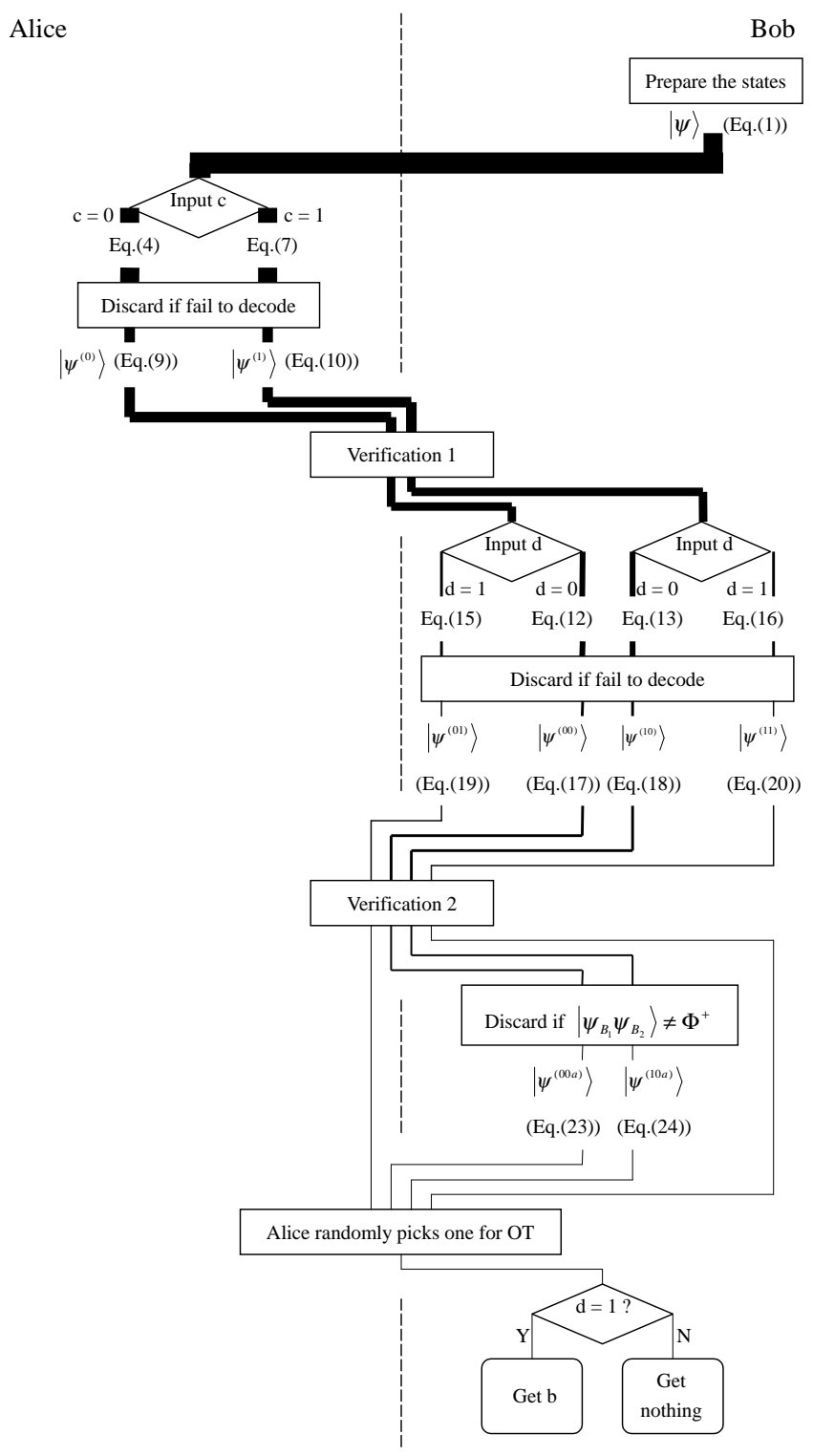

FIG. 1: A schematic flow chart of Protocol OT. The boxes on the left (right) represent the local operations on Alice's (Bob's) side, while the middle ones are those requiring collaboration of them. The width of the lines denote qualitatively the number of the states.
(I) For the state $|\psi\rangle$ given in Eq.(1), Alice cannot learn $q, r$ and $d$ simultaneously with the reliability $100 \%$, and Bob cannot learn $s$ and $c$ simultaneously with the reliability $100 \%$.

Proof: Let $\rho_{0}\left(\rho_{1}\right)$ denote the reduced density matrix of the quantum state on Alice's side corresponding to $q=q_{0}$ and $r=r_{0}\left(q \neq q_{0}\right.$ and $\left.r \neq r_{0}\right)$. To make sure that $q=q_{0}$ and $r=r_{0}$ simultaneously, Alice needs to distinguish $\rho_{0}$ from $\rho_{1}$. It can be proven that the optimal strategy for her to identify $\rho_{0}$ with the reliability $100 \%$ is to measure the states in the basis in which $\rho_{1}$ is diagonalized. Supposing that $\rho_{0}$ and $\rho_{1}$ are expressed in this basis with $\rho(k, l)$ denoting the element of the matrix $\rho$, the maximum probability for identifying $\rho_{0}$ is

$$
p_{0 \max }=\sum_{k \in\left\{k \mid \rho_{1}(k, k)=0\right\}} \rho_{0}(k, k) .
$$

When $|\psi\rangle$ takes the form as specified in Eq.(1), it is shown that $\left\{k \mid \rho_{1}(k, k)=0\right\}=\phi$ (the empty set) regardless of the values of $q$ and $r$. Therefore $p_{0 \text { max }}=0$, which means that Alice can never learn the exact values of $q$ and $r$ simultaneously with the reliability $100 \%$. Similarly, it can also be proven that Bob cannot learn $s$ and $c$ simultaneously with the reliability $100 \%$.

As for $d$, by comparing Eqs. (19) with (23) and Eqs.(20) with (24) respectively, we can see that after the step (6), the final states of systems $A_{1}$ and $A_{2}$ are exactly the same regardless Bob's choice of $d$. Therefore Alice cannot learn $d$ as long as the protocol can indeed force the participants to perform the honest measurement. This leads us to the next point of the proof.

(II) For the state $|\psi\rangle$, the steps (3-1) and (3-2) can force Alice to measure the states honestly in the step (2), and the step (5) can force Bob to measure the states honestly in the step (4).

Proof: Consider Alice's cheating first. Suppose that in the step (3-2), there are totally $\delta n^{\prime}$ sets of $|\psi\rangle$ which have not been measured by Alice honestly. Instead, she applies a minimal-error measurement or even delays the measurement. Then she does not know their $q$ or $r$ with the reliability $100 \%$, but only with a reliability being not larger than $\varepsilon$. As Bob picks randomly many $|\psi\rangle$ to check if Alice knows $q$ or $r$, the probability for Alice to pass the test is $\varepsilon^{O(\delta n)}$. Meanwhile, since only one $|\psi\rangle$ is randomly picked for the OT at the final stage, the probability for these $\delta$ sets of $|\psi\rangle$ to be picked is not greater than $\sum_{i=1}^{\min \left(\delta n^{\prime}, m\right)} C_{\delta n^{\prime}}^{i} C_{n^{\prime}-i}^{m-i} / C_{n^{\prime}}^{m}$. The order of magnitude of this probability is $O(\delta)$ as long as $n>>m$. Therefore the total probability for Alice to cheat successfully is bounded by $O(\delta) \varepsilon^{O(\delta n)}$, which can be made arbitrarily small as $n \rightarrow \infty$.

Thus Alice cannot use the minimal-error measurement, but has to use the measurements which always decode $q$ or $r$ with the reliability $100 \%$. Here it is shown that the honest measurement is the optimal one among all these measurements. Using the method described in (I), let $\rho_{0}$ and $\rho_{1}$ be the density matrices for $q=+$ and $\times$ 
respectively (being independent of $r$ ). In the Bell basis, both $\rho_{0}$ and $\rho_{1}$ are diagonalized. The maximum probabilities for Alice to identify them are the same: $p_{0 \max }=p_{1 \max }=1 / 2$, which can be reached simultaneously in the same measurement. Thus the maximum probability for Alice to decode $q$ with the reliability $100 \%$ is $p=\left(p_{0 \max }+p_{1 \max }\right) / 2=1 / 2$. And the operation in the step (2) is just the strategy that can reach this maximum. The calculation of the maximum probability for Alice to decode $r$ successfully is a little bit more complicated. In this case, $\rho_{0}$ (for $r=0$ ) and $\rho_{1}$ (for $r=1$ ) cannot be diagonalized simultaneously, and the maximum probability $p<\left(p_{0 \max }+p_{1 \max }\right) / 2$. But we can see that when Alice chooses to decode $r$ in the step (2), if the corresponding projection succeeds, she immediately gets 1 bit of information; while the projection fails, $\rho_{0}$ and $\rho_{1}$ collapse to the same density matrix, i.e., the upper bound of the average information that can be gained from the resultant final states is zero. This fact implies that Alice had already drawn as much information as possible from the states she received. Therefore when $r=0$ and $r=1$ occur with the same probability, the strategy in that step is exactly the optimal one for her to get $r$ with the reliability $100 \%$. The maximum probability of this procedure is also $p=1 / 2$. Namely, Alice cannot decode $q$ or $r$ unambiguously with a probability higher than that of the honest measurement.

As a result, if Alice makes her measurement without using the correct method in the step (2) or even delays her measurement until Bob announces which sets of $|\psi\rangle$ picked for the verification in the step (3-2), either she cannot reach the maximum efficiency such that she has to discard more data than what is allowed in the step (3-1), or there will inevitably be some undiscarded $q$ or $r$ whose reliability is only $\varepsilon<100 \%$. She cannot pass the test with a nontrivial probability, because in the step (3-2) it is no longer allowed to discard the data that she fails to decode. For this reason, Alice has to follow the protocol honestly.

Repeating the above procedure, we can obtain the similar result for the case in which Bob applies the minimal measurement or other dishonest measurements. Bob has to choose $d=0$ and $d=1$ with the specified ratio and use the method in the step (4) to measure all $\psi_{B_{1}} \psi_{B_{2}}$. Else he will only have a probability $O(\delta) \varepsilon^{O(\delta m)}$ to cheat without being caught.

From the above (I) and (II), we can see that the goal of OT can be achieved, as long as the initial state $|\psi\rangle$ takes the specific form given in Eq.(11). This allows us to proceed to the last but not the least part of the proof.

(III) Steps (3-3) and (5) are able to force Bob to prepare the states honestly.

Proof: The step (5) requires Bob to show that he has indeed input $d$ for all the remaining $|\psi\rangle$ (i.e., he already got $c$ or $s$ with the reliability $100 \%$ ), while only about $m / 2$ sets are allowed to be discarded. Therefore for the same reason in (II), in the step (4) the probability for Bob to get $c$ with the reliability $100 \%$ should reach $1 / 2$.
We shall prove that, if Bob does not prepare the initial states honestly, this probability will drop, or he will not pass the test in the step (3-3).

Let us first study what constrain will be put on the initial states by the step (3-3). There may exist many cheating strategies for Bob. But they can all be described by the following model. Bob sends Alice a quantum system $\alpha$ which is entangled with another system $\beta$. He performs any POVMs 22 on $\beta$ to get as much information as he can. A general form of the entangled system $\alpha \otimes \beta$ is

$$
|\psi\rangle=\sum_{k} f_{k}\left|\alpha_{k}\right\rangle\left|\beta_{k}\right\rangle
$$

Alice can check the partial density matrix $\rho_{\alpha}=$ $\sum_{k}\left\langle\beta_{k} \mid \psi\right\rangle\left\langle\psi \mid \beta_{k}\right\rangle$ of system $\alpha$ with her measurement in the step (3-3). Therefore to make Alice believe that he is honest, Bob has to prepare $\alpha \otimes \beta$ in such a way that $\rho_{\alpha}$ is much the same as that of $\psi_{A_{1}} \psi_{A_{2}}$ in the honest protocol. Thus for each single set of $|\psi\rangle$, the state of such a system can be expanded as 22]

$$
|\psi\rangle=\sum_{r \in\{0,1\}, q \in\{+, \times\}} f_{r, q}|r\rangle_{q}|r\rangle_{q}\left|B_{r, q}\right\rangle .
$$

Bob sends Alice the first two qubits, and keeps the last part on his side as $\beta$. Generally $\beta$ can include any systems at Bob's side and the environment, and even the systems $A_{1}$ and $A_{2}$ from other sets of $|\psi\rangle$ at Alice's side. But Bob does not know beforehand which $|\psi\rangle$ will be picked for the test in the step (3-3). So he needs to prepare $\beta$ with the following property: once the corresponding $|\psi\rangle$ is picked, he can always measure $\beta$ and get $q, r$ unmistakably. Thus $\beta$ has to contain the systems on Bob's side only, and all the states $\left|B_{r, q}\right\rangle$ with different $q, r$ need to be orthogonal to each other.

We now evaluate the amount of information on $c$ that Bob can obtain with such a state. Suppose that $|\psi\rangle$ eventually survives through the step (3). This state can be expressed as

$$
\begin{aligned}
|\psi\rangle= & \left(\sum_{r, q} f_{r, q} \Phi^{+}\left|B_{r, q}\right\rangle+\sum_{r}(-1)^{r} f_{r,+} \Phi^{-}\left|B_{r,+}\right\rangle\right. \\
& \left.+\sum_{r}(-1)^{r} f_{r, \times} \Psi^{+}\left|B_{r, \times}\right\rangle\right) / \sqrt{2}
\end{aligned}
$$

Since Alice already included this $|\psi\rangle$ in what she decoded with the reliability $100 \%$, if what she decoded is $q$, i. e., she has chosen $c=0$, she must have found $\Phi^{-}$or $\Psi^{+}$in her measurement. From this equation, we can see that the system $\beta$ must have collapsed into

$$
\left|B_{0}^{\prime}\right\rangle \equiv \sum_{r}(-1)^{r} f_{r,+}\left|B_{r,+}\right\rangle / \sqrt{\sum_{r} f_{r,+}^{2}}
$$

or

$$
\left|B_{1}^{\prime}\right\rangle \equiv \sum_{r}(-1)^{r} f_{r, \times}\left|B_{r, \times}\right\rangle / \sqrt{\sum_{r} f_{r, \times}^{2}}
$$


Similarly, if $c=1, \beta$ must have collapsed into

$$
\left|B_{0}^{\prime \prime}\right\rangle \equiv \sum_{q} f_{0, q}\left|B_{0, q}\right\rangle / \sqrt{\sum_{q} f_{0, q}^{2}}
$$

or

$$
\left|B_{1}^{\prime \prime}\right\rangle \equiv \sum_{q} f_{1, q}\left|B_{1, q}\right\rangle / \sqrt{\sum_{q} f_{1, q}^{2}} .
$$

Therefore if Bob can distinguish $\left\{\left|B_{k}^{\prime}\right\rangle\right\}$ from $\left\{\left|B_{k}^{\prime \prime}\right\rangle\right\}$, he knows Alice's choice of $c$. Define

$$
\rho_{0} \equiv \sum_{k}\left|B_{k}^{\prime}\right\rangle\left\langle B_{k}^{\prime}\right| / 2
$$

and

$$
\rho_{1} \equiv \sum_{k}\left|B_{k}^{\prime \prime}\right\rangle\left\langle B_{k}^{\prime \prime}\right| / 2
$$

The upper bound (Holevo bound) of the average information Bob can get is

$$
I_{a v}=S\left[\left(\rho_{0}+\rho_{1}\right) / 2\right]-\left[S\left(\rho_{0}\right)+S\left(\rho_{1}\right)\right] / 2,
$$

where the von Neumann entropy is $S(\rho)=-\operatorname{Tr}\left(\rho \log _{2} \rho\right)$ 23. From the symmetry of the equation, it can be seen that $I_{a v}$ will go to its extremum when Bob chooses $f_{r, q}=$ $1 / 2$ for all $r, q$. It is found that this extremum is the maximum. That is, if Bob prepares the initial state as

$$
|\psi\rangle=\sum_{r \in\{0,1\}, q \in\{+, \times\}}|r\rangle_{q}|r\rangle_{q}\left|B_{r, q}\right\rangle / 2,
$$

the probability for him to get $c$ with the reliability $100 \%$ will be maximized. In the previous paragraph, it is shown that all $\left|B_{r, q}\right\rangle$ need to be orthogonal. For illustration, it is natural to choose

$$
\left|B_{r, q}\right\rangle=|Q\rangle_{+}|r\rangle_{+}
$$

where $Q=0,1$ for $q=+, \times$. Then

$$
\left|B_{k}^{\prime}\right\rangle=|k\rangle_{+}|1\rangle_{\times}, \quad\left|B_{k}^{\prime \prime}\right\rangle=|0\rangle_{\times}|k\rangle_{+} \quad(k=0,1) .
$$

We can see that $\rho_{0}$ and $\rho_{1}$ are diagonalized simultaneously in the basis that Bob uses in the projection in the step (4). Therefore this projection is just the optimal strategy for Bob to decode $c$, and the maximum probability for the decoding to be successful is $1 / 2$. If Bob does not prepare the initial state in this way, this maximum probability cannot be reached as $I_{a v}$ is not optimized. Similar to the proof in (II), the probability for him to pass steps (5) can be made arbitrarily small as $m \rightarrow \infty$.

Combining points (I)-(III), we can conclude that the probability for Alice to know whether Bob gets $b$ or not (or the probability for Bob to get $b$ in more than $50 \%$ of the cases) is expressed as $O(\delta) \varepsilon^{O(\delta n)}$ (or $\left.O(\delta) \varepsilon^{O(\delta m)}\right)$, which is arbitrarily small by increasing $n, m$. Also, unlike the cheat sensitive protocols [24], the detection of cheating in our protocol will not cause the secret bit of OT to be revealed. As a result, the present Protocol OT is unconditionally secure. As our proof is based on the density matrices of the quantum states, rather than on a specific cheating strategy, our conclusion is general no matter what computational power the participants may have and what POVMs they may apply.

\section{RELATIONSHIP WITH THE NO-GO THEOREMS}

\section{A. The Lo's no-go theorem of quantum secure computations}

Though the above general proof of security against all possible cheating strategies seems complicated, the reason why this protocol can evade the cheating in the Lo's no-go theorem is clear. As mentioned in the introduction, the protocol does not satisfy the requirement (a) (Bob learns a prescribed function $f(i, j)$ unambiguously) in Ref. [19], on which the no-go proof is based. This is because Bob cannot learn the value of $b$ unambiguously in our protocol. Instead, he only learns $b$ with the probability $50 \%$. In the other $50 \%$ case, he has zero knowledge on $b$. In addition, rigorously speaking, the outcome of our protocol cannot be viewed as a prescribed function $f(i, j)$. The outcome depends not only on Alice's and Bob's inputs $i$ and $j$, but also on the quantum uncertainty in the measurement. For example, in the step (4) of our protocol, Bob's inputting $d=1$ does not mean that he can certainly obtain the value of $c$. Due to the quantum uncertainty in his measurement, he can only obtain $c$ successfully with the probability $50 \%$. As a result, the quantum state in our protocol is not the simultaneous eigenstate of different measurement operators that the participant uses for determining the parameters wanted by him (e.g., $s$ and $c$ ). He knows whether he gets a parameter successfully only if the measurement is performed. Then the state is disturbed, so that it cannot be used to get more parameters. Thus the protocol is secure against the cheating strategy in Ref.[19]. On the other hand, the definition of all-or-nothing OT only requires that at the end of the protocol, the two outcomes "Bob learns the value of $b$ " and "Bob has zero knowledge on $b$ " should occur with the equal probability $50 \%$; while it never requires that which outcome finally happens must be controlled only by the participants' inputs. Clearly, our protocol satisfies the rigorous definition of secure allor-nothing OT.

\section{B. The MLC no-go theorem of secure QBC}

Our result does not conflict with the MLC no-go theorem of secure QBC, because this no-go theorem does not apply directly to QOT (otherwise the Lo's no-go the- 
orem of quantum secure computation would be redundant). Let $P_{1}$ denote an all-or-nothing QOT protocol. Surely it does not implement QBC automatically. Instead, another protocol $P_{2}$ is needed, which makes use of the output of $P_{1}$ to accomplish QBC. The MLC no-go theorem reveals that the entire protocol $P_{1}+P_{2}$ cannot be secure. Then there are two possibilities: $P_{1}$ is insecure, or $P_{2}$ is insecure (if not both). But as we already proved rigorously in Sec. III, our all-or-nothing QOT protocol is unconditionally secure against any cheating strategy. Therefore the existence of the MLC no-go theorem implies that secure $P_{2}$ is impossible.

Indeed, though $\mathrm{BC}$ and $\mathrm{OT}$ are thought to be classically equivalent, "reductions and relations between classical cryptographic tasks need not necessarily apply to their quantum equivalents" 25]. So far there are two known methods to construct $P_{2}$ in classical cryptography, which all fail at the quantum level. One of the method is to repeat all-or-nothing OT many times [3]. More rigorously, according to Ref. [3], BC is realized by encoding the committed bit as $b=b_{1} \oplus b_{2} \oplus \ldots \oplus b_{k}$, and sending each $b_{i}$ from Alice to Bob through an all-or-nothing OT process. However, the resultant protocol is insecure because altering anyone of the $b_{i}$ can flip the value of the committed bit completely. Alice can simply execute the protocol honestly. If she wants to change the committed bit at the final stage, she simply announces one of the $b_{i}$ dishonestly. Since Bob knows $b_{i}$ at half of the cases only, Alice can cheat successfully with the probability $1 / 2$. Thus the scheme is broken. Another known method to realize BC from OT in classical cryptography is to build an 1-out-of-2 OT [26, 27] from all-or-nothing OT, and use the 1-out-of-2 OT to implement BC. But once again, it has to rely on the classical equivalence between 1-out-of-2 OT and all-or-nothing OT 26], which needs re-examination at the quantum level. As pointed out in Ref. 19], classical reduction would be applicable in quantum cryptography if a quantum protocol can be used as a "black box" primitive in building up more sophisticated protocols. However, we found recently [28] that the 1-out-of-2 OT protocol built upon the present quantum all-or-nothing OT protocol with the scenario developed in Ref. [26] is not rigorously a "black box" type quantum 1-out-of-2 OT specified in Ref. 19]. Especially, the inputs of the two participants are not independent of each other. Such a quantum 1-out-of-2 OT cannot be used to implement secure QBC with the method described in Ref. [29]. The reason lies in that the step (2) of the protocol described in Ref. 29] is inexecutable as Alice's input cannot be completed before Bob's input is entered. Thus the method also fails. Of course there may exist other methods to construct $P_{2}$, but due to the presence of the MLC no-go theorem, they are all bound to be insecure. In this sense, the classical reduction chain from OT to BC is broken in the present quantum case, and thus there exists no logic conflict between the present secure all-or-nothing QOT and the MLC no-go theorem of QBC.

\section{SUMMARY AND DISCUSSIONS}

In all, we proposed an quantum all-or-nothing oblivious transfer protocol based on quantum entangled states, and proved that it is unconditionally secure against any cheating strategy. It was also illustrated how the protocol evades the Lo's no-go theorem of the one-sided twoparty secure computation, as well as that the security of our QOT does not conflict with the MLC no-go theorem of QBC.

The existence of secure QOT protocol is important not only for multi-party protocols, but also for a better understanding of quantum theory. According to recent results [30], three fundamental information-theoretic constrains, namely, the impossibilities of (i) superluminal information transfer between two physical systems by performing measurements on one of them; (ii) broadcasting the information contained in unknown physical states; and (iii) unconditionally secure bit commitment, may suffice to entail that the observables and state space of a physical theory are quantum-mechanical. Therefore, clarifying the boundary between the capability and limitation of quantum cryptography, as well as the relationship between classical cryptography and its quantum counterpart, can certainly enrich our knowledge for searching the answer to Wheeler's query "Why the quantum" 30].

Finally, it is worth pinpointing that a QBC protocol somewhat similar to ours was proposed 31]: both protocols start with a 4-level system on Alice's side and rely on a verification procedure to avoid cheating. However, as pointed out by the authors, what they achieved in Ref.31 was merely an analog to OT, which does not meet the rigorous security requirement of the OT definition; in fact, they merely attempted to use the analog to realize a $\mathrm{QBC}$ protocol. In contrast, our protocol includes a further crucial verification on Bob's side, possessing at least three advantages: (i) the strict requirement of OT is met; (ii) the stand-alone security is proven to be unconditional; and (iii) it is convenient to modify ours to be a $p$-OT protocol [26].

We thank Hoi-Fung Chau and Hoi-Kwong Lo for their useful discussions. The work was supported by the RGC grant of Hong Kong (HKU7114/02P and HKU7045/05P).
[1] M. Rabin, technical report TR-81, Aiken Computation Laboratory, Harvard University, 1981

[2] S. Wiesner, SIGACT News, 15, 78 (1983).
[3] J. Kilian, in Proceedings of 1988 ACM Annual Symposium on Theory of Computing, May 1988, pp.20 (ACM, New York, 1988). 
[4] C. Crépeau, J. van de Graaf, and A. Tapp, in Advances in Cryptology: Proceedings of Crypto '95, Vol.963, pp.110 (Springer-Verlag, Berlin, 1995).

[5] P. W. Shor, in Proceedings of the 35th Annual Symposium on the Foundations of Computer Science, pp.124 (IEEE Computer Society, Los Alamitos, CA, 1994).

[6] C. H. Bennett, and G. Brassard, in Proceedings of IEEE International Conference on Computers, Systems, and Signal Processing, Bangalore, India, pp.175 (IEEE, New York, 1984).

[7] A. K. Ekert, Phys. Rev. Lett. 67, 661(1991); C. H. Bennett, ibid. 68, 3121 (1992); D. A. Meyer, ibid. 82, 1052 (1999).

[8] C. Crépeau and J. Kilian, in 29th Symp. on Found. of Computer Sci., pp. 42-52 (IEEE, 1988). C. H. Bennett, G. Brassard, C. Crépeau, and M. -H. Skubiszewska, in Advances in Cryptology: Proceedings of Crypto '91, Vol.576, pp.351 (Springer-Verlag, 1992).

[9] C. Crépeau, Journal of Modern Optics, 41, 2445 (1994).

[10] A. C. C. Yao, in Proceedings of the 26th Symposium on the Theory of Computing, 1995, pp.67 (ACM, New York, 1995).

[11] G. Brassard, C. Crepeau, R. Jozsa, and D. Langlois, in Proceedings of the 34th Annual IEEE Symposium on Foundations of Computer Science, 1993, pp.362 (IEEE, Los Alamitos, 1993).

[12] D. Mayers, Phys. Rev. Lett. 78, 3414 (1997); H.-K. Lo and H. F. Chau, Phys. Rev. Lett.78, 3410 (1997).

[13] G. Brassard, C. Crépeau, D. Mayers, L. Salvail, quant-ph/9712023

[14] D. A. Meyer, Phys. Rev. Lett. 82, 1052 (1999).

[15] S. J. van Enk, Phys. Rev. Lett. 84, 789 (2000).

[16] L. Goldenberg, L. Vaidman, and S. Wiesner, Phys. Rev. Lett. 82, 3356 (1999).

[17] C. Crepeau, and L. Salvail, in Advances in Cryptology:
Proceedings of Eurocrypt '95, pp.133 (Springer-Verlag, 1995).

[18] D. Mayers, and L. Salvail, in Proceedings of the Third Workshop on Physics and Computation-PhysComp '94, pp.69 (IEEE Computer Society Press, Dallas, 1994).

[19] H.-K. Lo, Phys. Rev. A, 56, 1154 (1997).

[20] H. F. Chau and H.-K. Lo, Fortsch. Phys. 46, 507 (1998).

[21] Here as well as in the steps (3-4), (5-1) and (5-3), a small deviation from the mean value of the equidistribution is tolerable. The range of tolerable deviation can be evaluated by the Bernshtein's law of large numbers and then agreed on by the participants. It can be proven that by choosing sufficiently large security numbers $n, m$ and the number of $|\psi\rangle$ picked for the verifications, the protocol can be successful with a probability larger than $1-x$, while the probability of the cheating via this deviation is less than $x$. Here $x$ can be made arbitrarily small.

[22] L. P. Hughston, R. Jozsa, and W. K. Wootters, Phys. Lett. A 183, 14 (1993).

[23] A. S. Holevo, Probabilistic and Statistical Aspects of Quantum Theory (North-Holland, Amsterdam, 1982).

[24] L. Hardy and A. Kent, Phys. Rev. Lett. 92, 157901 (2004).

[25] A. Kent, Phys. Rev. Lett. 90, 237901 (2003).

[26] C. Crépeau, in Advances in Cryptology: Proceedings of Crypto '87, Vol.293, pp. 350 (Springer-Verlag, 1988).

[27] S. Even, O. Goldreich, and A. Lempel, Advances in Cryptology: Proceedings of Crypto '82, pp.205 (Plenum 1982).

[28] G. P. He and Z. D. Wang, quant-ph/0504170

[29] T. Short, N. Gisin, and S. Popescu, quant-ph/0504134

[30] R. Clifton, J. Bub and H. Halvorson, Found. Phys. 33, 1561 (2003). H. Halvorson, J. Bub, quant-ph/0311065

[31] K. Shimizu, and N. Imoto, Phys. Rev. A 66, 052316 (2002); Phys. Rev. A 67, 034301 (2003). 